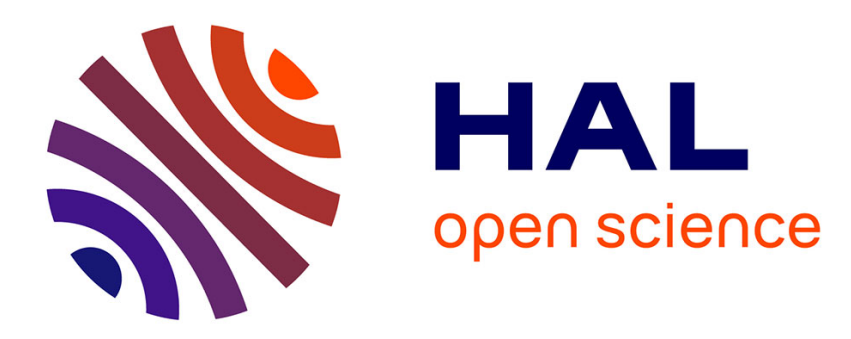

\title{
Thermosolutal natural convection in partially porous domains.
}

Dominique Gobin, Benoît Goyeau

\section{To cite this version:}

Dominique Gobin, Benoît Goyeau. Thermosolutal natural convection in partially porous domains.. Journal of Heat Transfer, 2012, Journal of Heat Transfer, 134 (3), pp.031013. 10.1115/1.4005147 . hal-00813377

\section{HAL Id: hal-00813377 \\ https://hal.science/hal-00813377}

Submitted on 10 Jul 2015

HAL is a multi-disciplinary open access archive for the deposit and dissemination of scientific research documents, whether they are published or not. The documents may come from teaching and research institutions in France or abroad, or from public or private research centers.
L'archive ouverte pluridisciplinaire HAL, est destinée au dépôt et à la diffusion de documents scientifiques de niveau recherche, publiés ou non, émanant des établissements d'enseignement et de recherche français ou étrangers, des laboratoires publics ou privés. 


\title{
THERMOSOLUTAL NATURAL CONVECTION IN PARTIALLY POROUS DOMAINS
}

\author{
Dominique Gobin* \\ Laboratoire FAST, CNRS \\ Université Pierre et Marie Curie \\ 91405 Orsay Cedex, France \\ Email: gobin@fast.u-psud.fr
}

\author{
Benoît Goyeau \\ Laboratoire EM2C, CNRS \\ École Centrale de Paris \\ 92295 Châtenay-Malabry Cedex, France \\ Email: benoit.goyeau@em2c.ecp.fr
}

\begin{abstract}
In many industrial processes or natural phenomena coupled heat and mass transfer and fluid flow take place in configurations combining a clear fluid and a porous medium. Since the pioneering work by Beavers and Joseph (1967), the modelling of such systems has been a controversial issue, essentially due to the description of the interface between the fluid and the porous domains. The validity of the so-called one-domain approach - more intuitive and numerically simpler to implement - compared to a two-domain description where the interface is explicitly accounted for, is now clearly assessed.

This paper reports recent developments and the current state of the art on this topic, concerning the numerical simulation of such flows as well as the stability studies. The continuity of the conservation equations between a fluid and a porous medium are examined and the conditions for a correct handling of the discontinuity of the macroscopic properties are analyzed. A particular class of problems dealing with thermal and double diffusive natural convection mechanisms in partially porous enclosures is presented, and it is shown that this configuration exhibits specific features in terms of the heat and mass transfer characteristics, depending on the properties of the porous domain. Concerning the stability analysis in a horizontal layer where a fluid layer lies on top of a porous medium, it is shown that the onset of convection is strongly influenced by the presence of the porous medium. The case of double diffusive
\end{abstract}

*Address all correspondence to this author. 
convection is presented in detail.

Keywords : Double diffusion - Fluid/porous layer - Stability analysis - Convection onset

\section{NOMENCLATURE}

$d^{*} \quad$ total thickness, $m$

$d^{* m} \quad$ thickness of the horizontal porous layer, $m$

$\widehat{d} \quad$ depth ratio $\left(\widehat{d}=d_{f}^{*} / d_{m}^{*}\right)$

$D_{f} \quad$ molecular diffusivity $m^{2} s^{-1}$

Da Darcy number $\left(D a=K / d^{* 2}\right)$

$g \quad$ gravity constant, $m s^{-2}$

$G r_{T}$ thermal Grashof number $\left(G r_{T}=g \beta_{T} \Delta T d^{* 3} / v^{2}\right)$

$G r_{S}$ solutal Grashof number $\left(G r_{S}=g \beta_{S} \Delta S d^{* 3} / v^{2}\right)$

$K \quad$ permeability of the porous medium, $\mathrm{m}^{2}$

$k \quad$ thermal conductivity, $W m^{-1} K^{-1}$

Le Lewis number $\left(L e=\alpha_{\mathrm{Tf}} D_{f}\right)$

$\operatorname{Pr} \quad$ Prandtl number $\left(\operatorname{Pr}=v / \alpha_{T f}\right)$

$R a_{T} \quad$ thermal Rayleigh number $\left(R a_{T}=G r_{T} \operatorname{Pr} D a\right)$

Sc Schmidt number $\left(S c=v / D_{f}\right)$

$T \quad$ temperature field $K$

$T_{1}\left(T_{2}\right)$ temperature of the upper or left (bottom or right) boundary, $K$

$S_{1}\left(S_{2}\right)$ concentration at the upper or left (bottom or right) boundary $\% w t .^{-1}$

V velocity field, $m s^{-1}$

$u(w)$ horizontal (vertical) velocity component, $m s^{-1}$

$x_{P}^{*} \quad$ thickness of the vertical porous layer, $m$

Greek symbols

$\alpha \quad$ slip coefficient 
$\alpha_{T} \quad$ thermal diffusivity, $m^{2} s^{-1}$

$\beta \quad$ stress jump coefficient

$\beta_{S} \quad$ solutal expansion coefficient, $\% w t^{-1}$

$\beta_{T} \quad$ thermal expansion coefficient, $K^{-1}$

$\varepsilon \quad$ porosity

$\varepsilon_{T} \quad$ ratio of diffusivities, $\alpha_{\mathrm{Tf}} / \alpha_{\mathrm{Tm}}$

$\kappa \quad$ dimensionless wave number

$\mu \quad$ dynamic viscosity of the fluid, $K g \mathrm{~m}^{-1} \mathrm{~s}^{-1}$

$v \quad$ kinematic viscosity of the fluid, $m^{2} s^{-1}$

$\rho \quad$ fluid density, $\mathrm{Kg} \mathrm{m}^{-3}$

$\sigma \quad$ growth rate

$\theta$ dimensionless temperature

$\phi \quad$ dimensionless concentration

Subscripts

$0 \quad$ reference

eff effective property

$f \quad$ fluid property

$m \quad$ porous medium property

\section{INTRODUCTION}

Processes involving heat and mass transfer and fluid flow in heterogeneous domains consisting of a porous layer with an adjacent fluid may be found in a great number of industrial applications (thermal insulation, filtration processes, storage of nuclear waste, drying processes, dendritic solidification, spreading on porous substrates, biofilm growth, gazeification of biomass, fuel cells ...) or in the context of environmental problems (geothermal systems, benthic boundary layers, ground- 
water pollution ...). In this context the problem of dealing with momentum conservation at a fluid-porous interface has been studied for many years. The simple situation of a Poiseuille flow in a partially porous channel, initiated by Beavers \& Joseph [1] followed by many other studies [2-4] allows to identify two classes of problems: 1 - the macroscopic model to be used in the porous domain, and 2 - the interface condition for tangential velocity and stress.

The present presentation deals more specifically with problems of natural convection in layers or cavities partially filled with a saturated porous medium. The problem has received some attention in the case of purely thermal convection, but we will more specifically focus on double diffusive problems which present specific features.

The purpose of this paper is first to discuss the different alternatives proposed in the literature to describe the conservation equations in such heterogeneous domains, basically the two domain formulation which considers the porous medium and the fluid as two distinct domains separated by a discrete interface where specific boundary conditions have to be explicitly written, versus the one domain formulation combining the terms for the porous and the fluid domains in a set of generalized conservation equations valid for both domains. In the second section of the paper, fluid flow and heat and mass transfer due to thermosolutal convection in a partially porous vertical enclosure are analyzed and it is shown that the presence of an even very thin porous layer has a strong influence on the behavior of this double diffusive system. Finally, both formulations are compared to study the stability problem at the onset of convection in an double diffusive horizontal layer where the fluid layer lies on top of the porous medium.

\section{MATHEMATICAL MODELLING}

Two different approaches are used for dealing with the fluid-porous interface treatment. The two-domain approach consists in writing the conservation equations in both the fluid and the porous regions with the associated interfacial boundary conditions. Generally, these boundary conditions often consider continuity of both the fields (temperature, concentration, velocity) and fluxes at the interface. However, as commented later in the present section, recent studies show that at the macroscopic scale, continuity is only obtained under particular conditions often related to the theoretical position of the interface (dividing surface) [3,5]. When this position is not known, jump boundary conditions could be considered depending on the physical nature of the problem under consideration [6]. In this context, the momentum transport at a fluid-porous interface deserves a special attention due to the fact that boundary conditions also depend on the macroscopic modeling in the porous region. In order to illustrate this problem, let us consider the one-dimensional fluid flow past a porous material studied by Beavers and Joseph [1]. The analytical solution for the flow is obtained by considering a Stokes flow while the 
momentum transport in the homogeneous porous layer is described by the Darcy law. Due to the difference in the order of the corresponding partial differential equations a semi-empirical slip boundary condition has been proposed [1]:

$$
\left.\frac{\partial u}{\partial z}\right|_{z=0}=\frac{\alpha}{\sqrt{K}}(u(0)-U(0))
$$

where $u$ and $U$ are the velocities in the fluid and porous regions, respectively, $K$ is the permeability of the homogeneous porous region and $\alpha$ represents a dimensionless slip coefficient which depends on the microstructure and location of the interface. When the Darcy-Brinkman equation is used in the porous layer [7], continuity of both velocity and shear stress can be imposed at the interface and the analytical solution for the Beavers and Joseph configuration gives $\alpha=\left(\mu_{\mathrm{eff}} / \mu_{\mathrm{f}}\right)^{-1 / 2}=$ $\varepsilon^{-1 / 2}$ where $\mu_{\mathrm{eff}}$ is the effective viscosity and $\varepsilon$ the porosity. Due to the domain of validity of the Brinkman correction, most of the studies devoted to the determination of the effective viscosity concern sparse porous structures or dilute suspensions for which the reduced viscosity is found to be close to the Einstein's law [8,9]. More recently, using a volume averaging method at the fluid-porous inter-region where the length-scale constraints are not satisfied, Ochoa-Tapia and Whitaker [10,11] derived a stress jump boundary condition such as:

$$
\left.\mu_{\mathrm{f}} \frac{\partial u}{\partial z}\right|_{z=0}-\left.\mu_{\mathrm{eff}} \frac{\partial U}{\partial z}\right|_{z=0}=\frac{\beta}{\sqrt{K}} u(0)
$$

where the stress jump coefficient $\beta$ is found to be on the order of one. For conciseness, the derivation of this boundary condition will not be presented in the present communicatior e references $[6,10,12]$ for details), nevertheless, its physical meaning deserves some comments. Indeed, it is important to recall that the jump condition given by Eq. (2) is actually an equivalent representation of momentum transport through the very thin interfacial region characterized by continuous spatial variations of the averaged properties (porosity, permeability) of the porous structure [8]. In fact, Eq. (2) results from the difference of the integration (over the inter-region) of the Generalized Transport Equation (GTE) and the integration of the transport equations in both homogeneous regions, respectively. Therefore, Eq. (2) applies at the so-called dividing surface

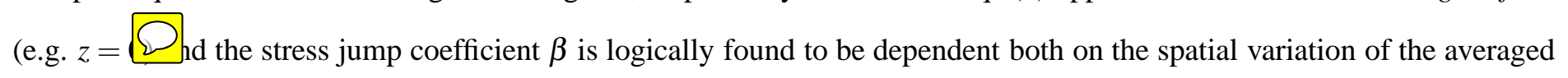
properties of the inter-region [3,6,12,13]. Let us recall that under its present form, Eq. (2) has been derived assuming continuity of the intrinsic average velocities. The relevance of this assumption has been addressed by using the matched asymptotic expansion method [3,5] and more recently in the context of volume averaging method [6]. Both analysis lead to general formulations involving jump conditions for the fields and the fluxes. However, depending on the physical problem, 
20. jump coefficients can be equal to zero and in that case continuity of fields and/or fluxes can be imposed. An alternative model to deal with the presence of fluid and porous regions is the one-domain approach. This latter considers the porous region as a pseudo-fluid and the whole domain is viewed as a continuum where the transition from the fluid to the porous regions is performed through spatial variations of properties [14]. Using this mathematical formulation avoids the explicit formulation of the boundary conditions at the fluid/porous interface. For this reason, the one-domain approach has been extensively used in numerical simulations or stability analysis of natural convection. Let us note that, in this approach, the derivatives of the macroscopic properties of the porous layer at the interface are distributional derivatives [15]. In that case, the one- and the two-domain approaches are shown to be equivalent and very good agreement is actually found when comparing the results obtained with both approaches $[16,17]$. An illustration of the equivalence between the one- and twodomain approaches is presented in section 4. Except for this case, in the forthcoming sections, the one-domain approach will be used for performing the simulations presented in this paper.

\section{THERMOSOLUTAL NATURAL CONVECTION IN A VERTICAL PARTIALLY POROUS CAVITY}

Among the problems involving natural convection in partially porous domains, we will tackle here the simulation of double diffusive convective flows in a binary fluid, confined in a vertical enclosure divided into sub-domains: a vertical porous layer and an adjacent fluid layer. This configuration may refer to many practical but complex situations (solidification of binary alloys, sewage treatment ...) and we will limit our interest in the present paper to very simple boundary conditions, typically imposed uniform temperatures and concentrations at the vertical walls.

Although a great number of studies have been dedicated to this geometry, the bibliography mostly refers to the case of thermal convection [18-20]. Our main focus in the present paper is to consider the case of double diffusive convection where the number of publications is more limited. Among them [21] most studies have been concerned with gaseous binary fluids for which the Prandtl and Lewis numbers on the order of 1 and thus extremely limited since the relevant features of double diffusion are linked to rather high Lewis numbers fluids $\left(L e \sim \mathscr{O}\left(10^{2}\right)\right.$ for liquids). The case of liquid metallic alloys $\left(L e \sim \mathscr{O}\left(10^{4}\right)\right)$ will not be examined here. We will analyze some specific features of double diffusive convection in such a partially porous enclosure, emphasizing the influence of the characteristics of the porous layer and the separate role of the double diffusive parameters. 


\subsection{Mathematical formulation}

In the two-dimensional rectangular cavity (height $\mathrm{H}$, total width $\mathrm{L}$ ) sketched in Figure 1, the porous layer (thickness $x_{\mathrm{P}}^{*}$ ) along the left wall is assumed to be homogeneous and isotropic. The porous medium is saturated by the binary fluid which fills the remaining of the enclosure. Different uniform temperatures and concentrations are specified at the external vertical walls of the cavity, and zero heat and species fluxes are assumed at the horizontal boundaries. The flow is assumed to be laminar and incompressible, and the binary fluid to be newtonian and to satisfy the linear Boussinesq approximation :

$$
\rho=\rho_{0}\left[1-\beta_{T}\left(T-T_{0}\right)-\beta_{S}\left(S-S_{0}\right)\right]
$$

Another classical assumption is to suppose that the porous matrix is in thermal equilibrium with the fluid, and that the Soret and Dufour effects are negligible. The macroscopic conservation equations both use the Darcy-Brinkman formulation in the porous layer and the Navier-Stokes equation in the binary fluid. The resulting mathematical model with the one-domain approach (the expression of the permeability is thus a prescribed function of space) leads to the following set of coupled equations in terms of the dimensionless variables defined in the nomenclature:

$$
\begin{gathered}
\nabla \cdot \mathbf{V}=0 \\
\frac{1}{\varepsilon}\left(\mathbf{V} \cdot \nabla \frac{\mathbf{V}}{\varepsilon}\right)=-\nabla P+\left(G r_{T} \theta+G r_{S} \phi\right) \mathbf{k}-\frac{1}{D a} \mathbf{V}+\frac{\mu_{\mathrm{eff}}}{\mu_{\mathrm{f}}} \nabla^{2} \mathbf{V} \\
\mathbf{V} . \nabla \theta=\frac{1}{P r} \nabla \cdot\left(\frac{k_{\mathrm{eff}}}{k_{\mathrm{f}}} \nabla \theta\right) \\
\mathbf{V} . \nabla \phi=\frac{1}{S c} \nabla \cdot\left(\frac{\mathscr{D}_{\mathrm{eff}}}{\mathscr{D}} \nabla \phi\right)
\end{gathered}
$$

At the boundaries, zero heat or species flux conditions are prescribed at the horizontal walls and Dirichlet boundary conditions at the vertical walls : $\theta=\phi=-0.5$ at the porous medium external wall $(x=0)$ and $\theta=\phi=0.5$ at the vertical wall in contact with the fluid $(x=1 / A)$.

The problem is characterized by the set of dimensionless parameters generally defined for double diffusive convection in fluids:

1. the thermal Rayleigh number defined with the fluid properties, $R a_{T}$, 
2. the buoyancy ratio $N=\beta_{S} \Delta S / \beta_{T} \Delta T$,

3. the Prandtl and Lewis numbers of the fluid, $\operatorname{Pr}$ and $L e$,

plus the parameters characterizing porous media:

4. the Darcy number (dimensionless permeability) of the porous layer, $D a$,

5. and geometrical parameters, the aspect ratio of the enclosure $A$, and the reduced thickness of the porous layer, $x_{P}=x_{P}^{*} / L$. $\mu_{\mathrm{f}}, k_{\mathrm{f}}$ and $\mathscr{D}_{\mathrm{f}}$ refer to the dynamic fluid viscosity, thermal conductivity and molecular diffusivity, respectively, while subscript 'eff' refers to the corresponding effective properties of the porous medium. The Nusselt and Sherwood numbers are the dimensionless average heat and mass fluxes along the vertical walls.

The set of equations (4-7) is solved using a standard finite volume procedure described elsewhere [22]. The method has been successfully used by the authors to solve heat and fluid flow problems in fluids and porous media in similar ranges of parameters. It has been first verified that thermal and thermosolutal natural convection results for $x_{\mathrm{P}} \rightarrow 1$ at any value of the Da number were in agreement with the standard Darcy-Brinkman version of the code and it has checked that at high values of the Darcy number the results at any value of $x_{\mathrm{P}}$ were identical to the results obtained for the pure fluid problem. The calculations in the non-homogeneous cavity have been compared for thermal convection against the existing results [19]. Depending on the permeability of the fluid layer, the strong temperature, concentration or velocity gradients may be located in the vicinity of the fluid-porous interface. Consequently, for low permeabilities compound meshes are used in order to limit the computational cost, and two distinct irregular (generally sinusoidal) horizontal grids are taken in the porous layer and in the fluid cavity. The number of nodes in each domain is a function of the Rayleigh numbers and of the thickness of the porous region. Typical values range between 145 and 252 nodes for the x-direction and from 202 up to 402 regularly spaced nodes in the z-direction, in order to solve the multicellular structures.

\subsection{Influence of the porous layer thickness and permeability}

The first sequence of results for a standard reference configuration $\left(R a_{T}=10^{6}, N=10, \operatorname{Pr}=10, \operatorname{Le}=100, A=2\right)$ shows the influence of the thickness and of the permeability of the porous layer. The results are displayed in terms of the Sherwood number (the average mass transfer) at the vertical walls (Figure 2). Three main features may be observed from the figure. First it can be seen that the presence of the porous layer has a significant influence on the mass transfer: if one recalls that the limit of high Darcy numbers $(D a=1)$ corresponds to a fully fluid cavity $\left(x_{\mathrm{P}}=0\right)$, it may be noted that a porous layer of any thickness drastically decreases the Sherwood number compared to the pure fluid case. For the present conditions a 
decrease by a factor 3 may be observed over the range of moderate permeabilities. The second feature is that the Sherwood number is practically unsensitive to the thickness of the porous layer over a large range of Darcy numbers from $D a \sim 10^{-6}$ to $D a \sim 10^{-2}$, corresponding to moderate and high permeabilities. Finally the influence of the porous layer thickness on mass transfer is essentially noticeable in the low permeability range $\left(D a<10^{-6}\right)$. In this range the mass transfer reduction is enhanced by the porous layer thickness.

These results are qualitatively the same for all the values of the Lewis number considered in the study (Le from 1 to 100) and they are in agreement with the observations made for heat transfer characteristics of fluid-porous cavities in purely thermal convection. If we now turn our attention to the average heat transfer variations as a function of permeability over the same range of $x_{\mathrm{P}}$ the curves displayed in Figure 3 show a quite different behavior. It can first be observed that the Nusselt number is not monotonically increasing with permeability. One can also note the strong sensitivity of the heat transfer characteristics to the porous layer thickness at all permeabilities in the $[0.1-0.8]$ range. Note that for thick porous layers $\left(x_{\mathrm{P}} \geq 0.8\right)$ the average Nusselt number remains very close to the pure conduction limit $(N u=A=2)$ over a wide range of Darcy numbers, indicating the absence of convective heat transfer in this range. This shows that the effect of the porous layer thickness on the boundary layers is not the same for species distribution and for the thermal field. It can be infered that this is due to the difference in diffusion lengths for temperature and concentration, that is to the Lewis number. We will now refine our analyis by the description of one particular case $\left(\operatorname{Ra} a_{T}=10^{6}, N=10, \operatorname{Pr}=10, L e=100, A=2\right.$ and $\left.x_{\mathrm{P}}=0.1\right)$ shown in white circles in Figs. 2 and 3. The evolution of the convective flow with increasing permeability results from the competition between two opposing effects. First, the flow penetration in the porous layer is easier at higher permeabilities and consequently the diffusive damping of the imposed temperature and concentration difference by the porous medium layer is smaller. The effective temperature and composition gradients governing the buoyancy forces are then expected to grow and the flow to be accelerated, resulting in higher heat and mass transfer. This effect may be observed on the Sherwood number. On the other hand, due to the difference between the thermal and species diffusivities $(L e=100)$, a central recirculation loop is driven at the scale of the thermal boundary layer thickness by a relatively smaller temperature difference. Locally the ratio of the buoyancy forces decreases, and the intensity of the internal thermal loop is decreasing, and also the average heat transfer. The process is thus dominated by the double diffusive mechanisms. The foregoing analysis is intended to refine the interpretation of this behavior in terms of the thermosolutal features of the solution. If we observe in detail the flow structure in the vicinity of the first Nusselt number minimum $\left(D a \sim 10^{-7}\right)$, the streamlines displayed at different values of the Darcy 
number (Figure 4) clearly show that the existence of a minimum is directly related to the flow structure. In the neighborhood of the minimum, a low velocity zone forms in the bottom part of the enclosure: this region is characterized by a stable vertical concentration gradient and the transverse (horizontal) heat transfer is mainly conductive [23,24]. So the decrease in the Nusselt is mainly due to the fact that the size of the main recirculation cell decreases. With increasing permeability the height of the stagnant zone increases, the local vertical concentration gradient decreases and the compositionally stratified "stagnant" zone gets destabilized by the lateral temperature gradient, resulting in the formation of a secondary co-rotative cell and a sudden increase of the Nusselt number. Then the formation process of a stagnant zone at the bottom of the enclosure resumes at $D a \sim 2 \times 10^{-7}$ with the related decrease in heat transfer, until the development of a third recirculation cell (not shown) allows for a new enhancement of the average heat transfer. Then, the increase in Darcy number results in a better penetration of the porous layer by the flow, until, at very high permeabilities a fully symmetrical tri-cellular structure characteristic of double diffusion in liquids is recovered.

\subsection{Influence of the double diffusive parameters}

As recalled above, specific features of double diffusive convection appear when the characteristic diffusion lengths for heat and solute are different. The parameter which governs the ratio between thermal and molecular diffusivity (the Lewis number) is currently on the order of $10^{2}$ or more, except for gases where $L e \sim 1$. In the latter case, the scale of heat and solute boundary layers are similar and the buoyancy forces have a merely additive effect. No multicellular structure is expected and the dependence of the Nusselt number with permeability follows the same variation as the Sherwood number, a smoothly monotonous increase, as displayed in Figure 2. We now consider the influence of the Lewis number over the range low and moderate values of $L e$ on the heat and mass transfer characteristics. All parameters are fixed, except for the mass diffusivity, meaning that the solutal Rayleigh number is increased in the same proportion as the Lewis number. The Sherwood number is uniformly increased with $L e$ (not shown) and we see (Fig. 5) that the Nusselt number variation is similar at low values $(L e \leq 5)$, and the heat transfer is decreasing as $L e$ increases, as expected from the scaling laws in the solutally dominated regime $(N>1)$ [25]. The Nusselt curve shows only one minimum for $L e=10$. It could be shown that the flow structure remains monocellular but the mechanism is similar to the second minimum of the reference case described above and the decrease in heat transfer is compensated around $D a=3 \times 10^{-5}$ by the penetration of the porous layer.

The other interesting feature to be analyzed is the influence of the buoyancy ratio. It is also well known that the double diffusive features of the flow are of mostly visible in the intermediate regime between the heat transfer dominated $(N<<1)$ 
and the mass transfer dominated $N>>1$ ) regimes, where both buoyancy forces are in competition. The numerical results presented here concern the cooperating case, that is, positive values of $N$ ranging from 1 to 10 for the same set of parameters, including $L e=100$ (Fig. 5). Again the Sherwood number (not shown) has the same qualitative behavior at different values of $N$ except that the increase in mass transfer occurs at smaller $D a$ for higher $N$. If we consider the $N u$ curves (Figure 6), there is almost no difference between $\beta_{S} \Delta S / \beta_{T} \Delta T=1$ or 2 , for which the Nusselt number variation is monotonous. A minimum may be noticed at $N=3$, but the limiting values (at $D a<10^{-7}$ or $D a>10^{-3}$ ) are also identical : here only the solutal Rayleigh number is modified through $N$ and it is known to have little influence on the Nusselt number. At $N=3$, one could show that the flow structure remains monocellular, a stagnant zone is formed at the bottom of the enclosure in the intermediate range of permeabilities, which results in a decrease of the average heat transfer. In this particular situation this zone remains stable, because the flow penetration of the porous layer accelerates the fluid before the stratified zone gets destabilized. For $N=5$, the flow structure exhibits three minima :

- the first minimum corresponds to the formation of a second cell,

- in the neighborhood of $\mathrm{Da}=10^{-5}$ a stagnant zone is formed below the second cell but this zone does not reach destabilization and the second cell increases again,

- around $D a=10^{-4}$ the flow penetration progressively increases the strength of the first cell and the second cell finally disappears,

- at high permeabilities the flow is mono-cellular.

The $N=5$ curve shows the transition between a globally monocellular flow structure at $N \leq 3$ and the 3 -cell structure at $N=10$.

As a conclusion to this section, it can be shown that the influence of a thin porous layer along a vertical wall of a rectangular enclosure has a remarkable influence on the heat and mass transfer transfers. Even if the conditions are significantly different from those met in real processes involving coupling between thermally and solutally driven flows, such as solidification processes, it seems to be important to carefully analyze those features to understand the coupled mechanisms and the heat and mass transfer characteristics. 


\section{ONSET OF THERMOSOLUTAL CONVECTION IN SUPERPOSED FLUID AND POROUS LAYERS}

Another class of studies dealing with transport phenomena at the interface between a fluid and a porous layer involves the onset of convection in heterogeneous horizontal layers. A large majority of stability analyses have been carried out using a two-domain approach, the conservation equations in the fluid and porous regions being coupled by interfacial boundary conditions. Indeed, for momentum transport, the studies uses Darcy's law in the porous region and Navier-Stokes equations in the fluid region [26-29]. In this situation, the coupling between the two homogeneous regions is obtained using a slip boundary condition [1] where the slip coefficient depends on the local nature and position of the interface [1]. In the case of thermal convection, the results using this modeling approach show a bimodal nature of the marginal stability curves where each mode corresponds to a different mode of convective instability [28]: at small wave numbers the convective flow starts in the "porous mode", while perturbations with large wave numbers are confined in the fluid layer. The alternative twodomain approach is based on the use of the Brinkman extension of Darcy's law which allows to satisfy the continuity of both velocity and stress at the fluid/porous interface $[7,30]$. It has been shown that the results obtained using the Beavers and Joseph condition [1] are in quantitative agreement depending on the value of the slip coefficient [31]. The volume averaging technique leads to a description of momentum transport conservation at the fluid/porous interface which implies a stress jump boundary condition $[10,11]$. This condition involves a jump coefficient $\beta$ which is explicitly dependent on the continuous spatial variations of the effective properties (porosity, permeability) at the fluid-porous inter-region $[3,8]$. In this section we will show that this latter model is in perfect agreement with the one-domain approach $[14,32]$. The objective of this section is to provide some results at the onset of thermosolutal natural convection in superposed fluid and porous layers with the one-domain model. The influence of some of the main parameters is also investigated.

\subsection{Mathematical model}

The configuration of interest in this section consists of an infinite horizontal porous layer (thickness $d_{\mathrm{m}}^{*}$ ) underlying a fluid layer of thickness $d_{\mathrm{f}}^{*}=d^{*}-d_{\mathrm{m}}^{*}$ (Fig. 7). The binary fluid is saturating the porous layer is assumed to be homogeneous and isotropic, newtonian and to satisfy the linear Boussinesq approximation. In the present analysis we assume that $\beta_{\mathrm{T}} \geq 0$ while $\beta_{\mathrm{S}} \leq 0$. The horizontal walls are impermeable and maintained at different temperatures and concentrations: $T_{1}, S_{1}$ (top) and $T_{2}, S_{2}$ (bottom). As in the previous section, the conservation equations are set into their dimensionless form using the following scales: $d^{*}$ for length, $d^{* 2} / v$ for time, $v / d^{*}$ for velocity, and $\left(\rho_{0} v^{2}\right) / d^{* 2}$ for pressure, where $v$ is the kinematic viscosity of the fluid. The temperature and concentration differences $\left(T-T_{0}\right)$ and $\left(S-S_{0}\right)$, are scaled by $\Delta T=T_{1}-T_{2}$ and 
$\Delta S=S_{1}-S_{2}$, respectively. $\mathbf{V}, \theta$ and $\phi$ represent the dimensionless velocity vector, the temperature and the concentration and the set of dimensionless conservation equations writes :

$$
\nabla \cdot \mathbf{V}=0
$$

$$
\begin{gathered}
\frac{\partial}{\partial t}\left(\frac{\mathbf{V}}{\varepsilon}\right)+\frac{1}{\varepsilon}\left(\mathbf{V} \cdot \nabla \frac{\mathbf{V}}{\varepsilon}\right)=\nabla \cdot\left(\frac{\mu_{\mathrm{eff}}}{\mu_{\mathrm{f}}} \nabla \mathbf{V}-P \mathbf{I}\right) \\
-\frac{1}{D a} \mathbf{V}+G r_{T} \theta \mathbf{k}+G r_{S} \phi \mathbf{k} \\
\frac{\partial \theta}{\partial t}+\mathbf{V} \cdot \nabla \theta=\frac{1}{P r_{\mathrm{f}}} \nabla \cdot\left(\frac{\alpha_{\mathrm{T}}}{\alpha_{\mathrm{Tf}}} \nabla \theta\right) \\
\varepsilon \frac{\partial \phi}{\partial t}+\mathbf{V} \cdot \nabla \phi=\frac{1}{S c_{\mathrm{f}}} \nabla \cdot\left(\frac{\mathscr{D}_{\mathrm{eff}}}{\mathscr{D}} \nabla \phi\right)
\end{gathered}
$$

These equations introduce the usual set of dimensionless parameters characterizing double diffusive convection the thermal and solutal Grashof numbers based on $d^{*}$ and the fluid Prandtl and Schmidt numbers. In the solute transport equation (11), due to the absence of solute diffusion in the solid phase, the effective solute diffusion coefficient in the absence of dispersion effect has been taken such as $D_{\text {eff }}=\varepsilon D_{\mathrm{f}}(\varepsilon=$ porosity). In the momentum equation (9) the dimensionless permeability $D a$ is the Darcy number while the reduced viscosity in the Brinkman term has be taken such as $\mu_{\mathrm{eff}} / \mu_{\mathrm{f}}=1 / \varepsilon$ [33]. The second Brinkman correction term has been neglected. Finally, $\alpha_{T}$ in equation (10) is the thermal diffusivity $\left(\alpha_{T}=\alpha_{\mathrm{Tf}}\right.$ in the fluid and $\alpha_{T}=\alpha_{\mathrm{Tm}}$ in the porous region). The associated dimensionless boundary conditions at the external walls are: $\mathbf{V}(1)=\mathbf{V}(0)=\mathbf{0} ; \theta(1)=T_{1}-T_{0} / \Delta T ; \theta(0)=T_{2}-T_{0} / \Delta T ; \phi(1)=S_{1}-S_{0} / \Delta S ; \phi(0)=S_{2}-S_{0} / \Delta S$. Let us mention that the effective properties $\left(\varepsilon, D a\right.$, and $\left.\alpha_{T}\right)$ in equations (8)-(11) are Heaviside functions and therefore their differentiation must use distributional derivatives $[15,34]$. The perturbation equations are obtained using the classical general decomposition. The basic state is assumed to be quiescent with linear functions of $\mathrm{z}$ for the temperature and concentration fields. The linearized system is then written in terms of the following expression for the vertical velocity component, the temperature and concentration:

$$
\left(w^{\prime}, \theta^{\prime}, \phi^{\prime}\right)=(W(z), \Theta(z), \Phi(z)) e^{i \kappa x+\sigma t}
$$

$\kappa$ is the dimensionless wave number and $\sigma$ is the complex growth rate. Assuming the principle of exchange of stability $(\sigma=0)$ the resulting eigenvalue problem is then solved using the Generalized Integral Transform Technique (GITT) [35,36]. The critical Grashof is obtained by minimization over $\kappa$ (see $[31,37]$ for details). This method has been validated by comparison with the exact values obtained in full fluid and porous cavities [38]. 


\subsection{Comparison of the one-domain and the two-domain approaches}

The first feature to be shown in this section is the equivalence of the one- and two-domain approaches. The corresponding stability analysis conducted using the two-domain approach $(2 \Omega)$ leads to write the conservation equations in each domain, and to specify the appropriate interface conditions. Let us remind that in order to be consistent with the $1 \Omega$ model, the momentum conservation is described by the Navier-Stokes in the fluid and by the Darcy-Brinkman formulation in the porous layer. The comparison is made in the case of thermal convection and the marginal stability curves obtained with both approaches, that is, the presentation of the critical thermal Rayleigh number $R a_{T}$ at the onset of convection versus the wave

number $\kappa$ for different values of the depth ratio $(\widehat{d})$. The main feature of the $2 \Omega$ model is the explicit expression of the interface conditions, which in dimensionless terms write, for the thermal convection case:

$$
\begin{aligned}
& \theta_{\mathrm{f}}=\theta_{\mathrm{m}} \quad \frac{\partial \theta_{\mathrm{f}}}{\partial z}=\frac{1}{\varepsilon_{T}} \frac{\partial \theta_{\mathrm{m}}}{\partial z} \\
& \mathbf{V}_{\mathrm{f}}=\mathbf{V}_{\mathrm{m}} \quad \frac{\partial u_{\mathrm{f}}}{\partial z}=\frac{1}{\varepsilon} \frac{\partial u_{\mathrm{m}}}{\partial z} \\
& -P_{\mathrm{f}}+2 \frac{\partial w_{\mathrm{f}}}{\partial z}=-P_{\mathrm{m}}+\frac{2}{\varepsilon} \frac{\partial w_{\mathrm{m}}}{\partial z}
\end{aligned}
$$

where $\varepsilon_{T}=\alpha_{\mathrm{T}_{\mathrm{f}}} / \alpha_{\mathrm{T}_{\mathrm{m}}}$. The results presented in Fig. (8) show a perfect agreement between the $1 \Omega$ and the $2 \Omega_{D B}$ approaches. Indeed, both formulation lead to the same bimodal behavior whatever the thickness ratio $\widehat{d}$. Each mode corresponds to a different structure of the convective flow. Indeed, at small wave numbers $(\kappa \sim 2.5)$ the convective flow involves the whole cavity ("porous mode") while for large wave number perturbations the convective flow is mainly confined in the fluid layer ("fluid mode"). A transition between the porous mode and the fluid mode is observed at $\widehat{d}=0.14$.

\subsection{Influence of the jump coefficient.}

It has been stressed in section 2 that the condition of tangential stress continuity (eq. 14) is a particular case of the general interface condition (2) with $\beta=0$. The influence of the stress jump coefficient $\beta$ on the stability of the system is thus of interest. In the case of purely thermal convection again, this influence is presented in Fig. 9 in terms of the critical thermal Rayleigh number $G r_{\mathrm{T}} P r_{\mathrm{f}} D a$ for $\widehat{d}=0.10, D a=10^{-5}$ and $\varepsilon=0.39$. The figure shows that the bimodal nature of the stability curve is not influenced by the value of $\beta$. It may be seen that the "porous mode" obtained at small wave numbers 
$(\kappa \sim 2.5)$ where the convective flow involves the whole layer thickness is unchanged with beta, while increasing the stress jump coefficient strongly modifies the fluid mode inducing a more unstable situation at larger wave numbers. This is due to the fact that the jump condition (2) actually takes into account the spatial variation of the permeability in the interfacial region leading to an increase of the velocity in the vicinity of the interface $[3,8]$.

Similar conclusions may be drawn for higher depth ratios. The reduction of the critical Rayleigh number is also coupled to a decrease of the critical wave number of the fluid mode (see Table 1). Finally, the influence of the stress jump coefficient depends on the magnitude of the Darcy number. For small values of $D a$ (not shown) or for very large values of the dimensionless permeability $(D a \rightarrow \infty)$, the stress coefficient hardly influences the marginal stability curves. For small Darcy numbers the porous layer tends towards a solid wall and a no-slip condition holds at the interface while for $D a \rightarrow \infty$ the porous media behaves as a fluid, and in that case, the interface disappears. For intermediate values of the permeability, it is shown that the influence of the jump coefficient on the marginal stability curves may be important. These results clearly illustrate the importance of the fluid-porous interfacial modelling. This is particularly true for very irregular interfaces where the complexity of the microstructure has to be taken into account. As previously noted, the jump coefficient $\beta$ can be related to the spatial variations of the average properties of the interfacial region (mesoscopic representation). However, a more accurate description would consist, for a given interfacial microstructure, in determining the jump coefficient by assessing and solving the local closure problems associated to the derivation of the jump boundary condition.

\subsection{Onset of thermosolutal convection}

This section is dedicated to the problem of double diffusive convection in horizontal fluid-porous layers. In a first step the solution procedure has been validated by comparing the numerical results to the exact values for the Rayleigh-Bénard problem both in a pure fluid $(D a \rightarrow \infty, \varepsilon=1)$ and in a full porous layer $\left(\widehat{d}=d_{\mathrm{f}}^{*} / d_{\mathrm{m}}^{*} \rightarrow 0\right)$. As expected, in both cases, the marginal stability curves plotted in the $\left(R a_{T}, R a_{S}\right)$ plane are straight lines [39] $\left(R a_{T}=G r_{T} P r_{\mathrm{f}} D a\right.$ and $\left.R a_{S}=G r_{S} S c_{\mathrm{f}} D a\right)$. According to the boundary conditions given in section 4.1 , the fluid case leads to $-R a_{\mathrm{Tf}}+R a_{\mathrm{Sf}}=1707.77$ with an associated wave number $\kappa=3.12$. The full porous configuration leads to $-R a_{\mathrm{Tm}}+R a_{\mathrm{Sm}}=4 \pi^{2} \approx 39.48$, with $\kappa=\pi \approx 3.14$. From the definitions $\Delta T=T_{1}-T_{2}$ and $\Delta S=S_{1}-S_{2}$, it is possible to identify the regions where the temperature and concentration gradients are stabilizing or destabilizing. One verifies that the curves $R a_{S} \times R a_{T}$ obtained with the present analysis are straight lines intersecting the axes at $(0,1707.77) ;(-1707.77,0)$ and $(0,39.48) ;(-39.48,0)$. Unless otherwise specified, all the results presented here have been obtained with the following parameters: $\operatorname{Pr}_{\mathrm{f}}=10, D a=10^{-5}, \varepsilon=0.39$, and 
$\varepsilon_{T}=\alpha_{\mathrm{Tf}} / \alpha_{\mathrm{Tm}}=1$. Figure 10 shows the stability curves obtained for different values of the depth ratio $\widehat{d} . R a_{\mathrm{T}}=0$ and $R a_{\mathrm{S}}=0$ correspond to the pure solutal or thermal cases, respectively. It is shown that as $R a_{\mathrm{T}}$ increases (the thermal buoyancy forces are more stabilizing) higher concentration gradients are needed to destabilize the system. Contrarily to the above limiting cases, the evolution of the stability curves are not straight lines for $R a_{\mathrm{T}} \geq 0$. This change can be explained by representing the critical wave number $\kappa$ as a function of the thermal Rayleigh number $R a_{\mathrm{T}}$ (Figure 11). Two regions are clearly identified: for negative values of $R a_{\mathrm{T}}$, the critical mode corresponds to convection cells at large wave numbers while the critical mode for $R a_{\mathrm{T}} \geq 0$ corresponds to cells at small wave numbers. These results indicate that the bimodal nature of the marginal stability curves obtained for the pure thermal convection case $[28,36]$ is also present in the thermosolutal case.

At small wave numbers the convective flow takes place in the whole cavity ("porous mode") while perturbations of large wave numbers lead to a convective flow mainly confined in the fluid layer ("fluid mode"). The streamline patterns and the vertical velocity profiles for $\widehat{d}=0.8$ and different values of $R a_{\mathrm{T}}$ are presented in Figures 12a to 12d. Contrarily to the pure thermal case where a single convection cell is observed, the onset of the thermosolutal case is characterized by a multi-layer regime for $R a_{\mathrm{T}} \ll 0$. This change is illustrated in Figures $12 \mathrm{a}$ and $12 \mathrm{~b}$. Indeed, for $R a_{T}=-20$ (Figure 12a), three contrarotating cells are present in the fluid region while the velocity field in the porous region is close to zero. When $R a_{\mathrm{T}}$ increases, the wave length of the cell also increases (the wave number decreases) and at $R a_{T}=0$ (Figure $12 \mathrm{~b}$ ) a monocellular flow is obtained (pure solutal case). For $R a_{T}=+20$ (Figure 12c) the flow starts to penetrate in the porous medium to finally occupy more or less the whole cavity for $R a_{T}=+50$ (Figure 12d). Note that the multi-cellular regime of the thermosolutal case, had been already observed by Chen \& Chen [28] using a two-domain approach. Zhao \& Chen [32] also have observed this type of multi-cellular structures using a one-domain model but they were not able to capture the two convective modes. It seems to us that one of the possible origin of this difference lies in the fact that the differentiation of the discontinuous functions at the interface was not taken in the sense of distributions. In addition, in both studies, only one value of the thermal Rayleigh number was considered $\left(R a_{\mathrm{T}} \sim 50\right)$. Finally, the influence of the thermal diffusivity ratio: for $\widehat{d}=0.8$ is presented in Figure 13. It is shown that a lower value of $\varepsilon_{\mathrm{T}}$ leads to a more unstable situation whatever the thermal Rayleigh number. As expected, in the absence of thermal buoyancy forces $\left(R a_{\mathrm{T}}=0\right) \varepsilon_{\mathrm{T}}$ has no influence on the stability of the system. Moreover, it can be seen that the differences between the curves obtained for $\varepsilon_{T}=0.7$ and 1 increases with $\left|R a_{\mathrm{T}}\right|$. The marginal stability curves ( $R a_{\mathrm{S}}$ versus $\kappa$ ) for two different values of $R a_{\mathrm{T}}$ have been computed (not shown). For $R a_{T}=-20$ (the temperature gradient is destabilizing) and $\varepsilon_{T}=1$, it is important to remember that the convective flow is confined in the 
fluid region (see streamline patterns on Figure 12a). For $\varepsilon_{T}<1$ heat diffusion is easier in the porous medium and therefore the temperature at the interface increases. Under these circumstances, the temperature gradients in the fluid become more important, giving rise to a smaller value of the critical solutal Rayleigh number. On the other hand, for $R a_{T}=20$ it is shown that the critical mode is obtained for small wave numbers ("porous mode"). Decreasing $\varepsilon_{\mathrm{T}}$ hardly destabilizes the "porous mode" while the "fluid mode" (large wave numbers) is found to be more stable. This behaviour can be explained by the stabilizing effect of the temperature gradient.

\section{CONCLUSION}

This note recalls the main results concerning the problem of double diffusive convection in partially porous domains, and the interaction between heat and mass transfer and fluid flow in a fluid and a porous layer. It had been seen that the discussion raised by the formulation of the problem is now clarified, in the limit of validity of the Darcy-Brinkman macroscopic description. A specific behavior of the heat and mass transfer characteristics and its dependance upon the double diffusive parameters has been explored in the case of vertical layers. The consequences on the stability problem in horizontal layers have been analyzed and the influence of the model parameters have been assessed.

The limitation of the study lies in the choice of very simple boundary conditions at the external walls and only general trends may be drawn in this limited frame. More efforts are requested for the solution of more specific problems. In particular many fields of applications deal with processes where these convective problems are coupled with phase change mechanisms, such as in solidification processes [40-43] or in environmental problems at the interface between seawater and under-ice melt ponds in the arctic polar ocean $[44,45]$. Such problems are presently under study as developments of the studies presented above.

\section{Acknowledgment}

The authors gratefully acknowledge the contribution of their PhD students, Silvia Hirata, Adrian Neculae and Jean-Pierre Songbe.

\section{REFERENCES}

[1] Beavers, G. S., and Joseph, D. D., 1967. "Boundary conditions at a naturally permeable wall". J. Fluid Mech., 30, pp. 197-207. 
[2] Chang, M., 2006. "Thermal convection in superposed fluid and porous layers subjected to a plane poiseuille flow". Physics of Fluids, 18(3), pp. 1-7.

[3] Chandesris, M., and Jamet, D., 2006. "Boundary conditions at a planar fluid-porous interface for a poiseuille flow". Int. J. Heat Mass Trans., 49, pp. 2137-2150.

[4] Hill, A. A., and Straughan, B., 2008. "Poiseuille flow in a fluid overlying a porous medium". J. Fluid Mech., 603, pp. 137-149.

[5] Chandesris, M., and Jamet, D., 2009. "Jump conditions and surface-excess quantities at a fluid/porous interface: a multi-scale approach”. Trans. Porous Med., 78(3), pp. 403-418.

[6] Valdés-Parada, F. J., Alvarez-Ramrez, J., Goyeau, B., and Ochoa-Tapia, J. A., 2009. “Computation of jump coefficients for momentum transfer between a porous medium and a fluid using a closed generalized transfer equation". Trans. Porous Med., 78, pp. 439-457.

[7] Neale, G., and Nader, W., 1974. "Practical significance of Brinkman's extension of Darcy’s law : Coupled parallel flow within a channel and a bounding porous medium". The Canadian J. of Chem. Eng., 52, pp. 475-478.

[8] Goyeau, B., Lhuillier, D., Gobin, D., and Velarde, M. G., 2003. "Momentum transport at a fluid-porous interface". Int. J. Heat Mass Trans., 46(21), pp. 4071-4081.

[9] Einstein, A., 1906. "Eine neue Bestimmung der Moleküldimensionen”. Annalen der Physik, 19(4), pp. $289-306$.

[10] Ochoa-Tapia, J. A., and Whitaker, S., 1995a. "Momentum transfer at the boundary between a porous medium and a homogeneous fluid-I. Theoretical development". Int. J. Heat Mass Trans., 38, pp. 2635-2646.

[11] Ochoa-Tapia, J. A., and Whitaker, S., 1995b. "Momentum transfer at the boundary between a porous medium and a homogeneous fluid-II. Comparison with experiment”. Int. J. Heat Mass Trans., 38, pp. 2647-2655.

[12] Valdés-Parada, F. J., Goyeau, B., and Ochoa-Tapia, J. A., 2007. “Jump momentum boundary condition at a fluid-porous dividing surface: Derivation of the closure problem”. Chemical Engineering Science, 62, pp. 4025-4039.

[13] Chandesris, M., and Jamet, D., 2007. "Boundary conditions at a fluid-porous interface: An a priori estimation of the stress jump coefficients". Int. J. Heat Mass Trans., 50, pp. 3422-3436.

[14] Arquis, E., and Caltagirone, J., 1984. "Sur les conditions hydrodynamiques au voisinage d'une interface milieu fluidemilieu poreux : application à la convection naturelle”. C. R. Acad. Sc., 299-II, pp. 1-4.

[15] Kataoka, I., 1986. "Local instant formulation of two-phase flow". Int. J. Multiphase Flow, 12(5), pp. 745-758. 
[16] Hirata, S. C., Goyeau, B., Gobin, D., Chandesris, M., and Jamet, D., 2009. "Stability of natural convection in superposed fluid and porous layers: equivalence of the one- and two-domain approaches". Int. J. Heat Mass Trans., 52(1-2), pp. 533-536.

[17] Jamet, D., Chandesris, M., and Goyeau, B., 2009. "On the equivalence of the discontinuous one- and two-domain approaches". Trans. Porous Med., 78, p. 403418.

[18] Beckermann, C., Ramadhyani, S., and Viskanta, R., 1987. "Natural convection flow and heat transfer between a fluid layer and a porous layer inside a rectangular enclosure". J. Heat Trans., 109, pp. 363-370.

[19] LeBreton, P., Caltagirone, J. P., and Arquis, E., 1991. "Natural convection in a square cavity with thin porous layers on its vertical walls". J. Heat Trans., 113, pp. 892-898.

[20] Mercier, J., Weisman, C., Firdaous, M., and LeQuéré, P., 2002. "Heat transfer associated to natural convection flow in a partly porous cavity”. J. Heat Trans., 124, pp. 130-143.

[21] Akbal, S., and Baytas, F., 2008. "Effects of non-uniform porosity on double diffusive natural convection in a porous cavity with partially permeable wall”. Int. J. Thermal Sci., 47(7), pp. 875-885.

[22] Gobin, D., Goyeau, B., and Songbe, J., 1998. "Double diffusive natural convection in a composite fluid-porous layer". J. Heat Trans., 120, pp. 234-242.

[23] Bennacer, R., and Gobin, D., 1996. "Cooperating thermosolutal convection in enclosures: scale analysis and mass transfer". Int. J. Heat Mass Trans., 39(13), pp. 2671-2681.

[24] Gobin, D., and Bennacer, R., 1996. "Cooperating thermosolutal convection in enclosures: heat transfer and flow structure.”. Int. J. Heat Mass Trans., 39(13), pp. 2683-2697.

[25] Bejan, A., and Khair, K. R., 1985. "Heat and mass transfer by natural convection in a porous medium". Int. J. Heat Mass Trans., 28(5), pp. 909-918.

[26] Nield, D. A., 1977. "Onset of convection in a fluid layer overlying a layer of a porous medium". J. Fluid Mech., 81, pp. 513-522.

[27] Nield, D. A., 1983. "The boundary correction for the Rayleigh-Darcy problem limitations of the Brinkman equation". J. Fluid Mech., 128, pp. 37-46.

[28] Chen, F., and Chen, C. F., 1988. "Onset of finger convection in a horizontal porous layer underlying a fluid layer". $J$. Heat Trans., 110, pp. 403-409. 
[29] Carr, M., and Straughan, B., 2003. "Penetrative convection in a fluid overlying a porous layer". Advances in Water Resources, 26(3), pp. 263-276.

[30] Brinkman, H. C., 1947. "A calculation of the viscous force exerted by flowing fluid on a dense swarm of particles". Appl. Sci. Res., A1, pp. 27-34.

[31] Hirata, S. C., Goyeau, B., Gobin, D., Carr, M., and Cotta, R. M., 2007. "Stability analysis of natural convection in adjacent fluid and porous layer: influence of interfacial modeling”. Int. J. Heat Mass Trans., 50(7-8), pp. 1356-1367.

[32] Zhao, P., and Chen, C. F., 2001. "Stability analysis of double-diffusive convection in superposed fluid and porous layers using a one-equation model". Int. J. Heat Mass Trans., 44, pp. 4625-4633.

[33] Whitaker, S., 1999. The method of volume averaging, Vol. 13. Kluwer Academic Publishers.

[34] Schwartz, L., 1961. Méthodes mathématiques pour les sciences physiques. Hermann, Paris.

[35] Cotta, R. M., 1993. Integral Transforms in Computational Heat and Fluid Flow. CRC Press, Boca Raton, FL.

[36] Hirata, S. C., Goyeau, B., Gobin, D., and Cotta, R. M., 2006. "Stability in natural convection in superposed fluid and porous layers using integral transforms". Num. Heat Trans., 50(5), pp. 409-424.

[37] Hirata, S. C., Goyeau, B., and Gobin, D., 2007. "Stability of natural convection in superposed fluid and porous layer: influence of the interfacial jump boundary condition". Physics of Fluids, 19, p. 058102.

[38] Chandrasekhar, S., 1961. Hydrodynamic and Hydromagnetic Stability. Oxford University Press, London.

[39] Nield, D. A., and Bejan, A., 1999. Convection in porous media. Springer-Verlag New York Inc.

[40] Amberg, G., and Homsy, G., 1993. "Nonlinear analysis of buoyant convection in binary solidification with application with channel formation". J. Fluid Mech., 252, pp. 79-98.

[41] Worster, M. G., 1997. "Convection in mushy layers". Ann. Rev. Fluid Mech., 29, pp. 91-122.

[42] Worster, M., 2000. Solidification of fluids. CU

[43] Guba, P., and Worster, M., 2006. "Free convection in laterally solidifying mushy regions". J. Fluid Mech., 558, pp. 69-78.

[44] Alexandrov, D., and Vizovtseva, I., 2008. "To the theory of underwater ice evolution, or nonlinear dynamics of false bottoms".. Int. J. Heat Mass Trans., 51(21), pp. 5204-5208.

[45] Tatarniuk, C., Donahue, R., and D., S., 2009. "Freeze separation of salt contaminated melt water and sand wash water at snow storage and sand recycling facilities". Cold Regions Science and Technology, 57, pp. 61-66. 


\section{Figure Captions}

Figure 1. Schematic description of the problem.

Figure 2. Mass transfer variation with permeability and porous layer thickness $\left(R a_{T}=10^{6}-N=10-\operatorname{Pr}=10-A=2\right)$

Figure 3. Heat transfer variation with permeability and porous layer thickness $\left(R a_{T}=10^{6}-N=10-\operatorname{Pr}=10-A=2-L e=\right.$ 100)

Figure 4. Heat transfer and flow structure around the first minimum: $5 * 10^{-8}<D a<5 * 10^{-7} ; \Delta \psi=0.1\left(R a_{T}=10^{6}\right.$, $N=10, x_{p}=0.1, L e=100, \operatorname{Pr}=10, A=2$ ). For comparing the flow structures, the streamlines have been plotted using the maximum value of the $\psi_{\max }$ for all cases $(\psi=0$ at the walls and $\Delta \psi=0.1)$.

Figure 5. Heat transfer variation with permeability for different Lewis numbers $\left(R a_{T}=10^{6}-N=10-x_{\mathrm{P}}=0.1-\operatorname{Pr}=10-\right.$ $A=2)$

Figure 6. Heat transfer variation with permeability for different buoyancy ratios $\left(R a_{T}=10^{6}-x_{\mathrm{P}}=0.1-\operatorname{Pr}=10-L e=100\right.$ $-A=2)$

Figure 7. Geometric configuration of the problem.

Figure 8. Marginal stability curves : comparison between the $1 \Omega$ and the $2 \Omega_{D B}$ approaches for $\widehat{d}=d_{f}^{*} / d_{m}^{*}=0.08$ and $\widehat{d}=0.10\left(D a=7.4410^{-6}\right.$.

Figure 9. Influence of the stress jump coefficient $\beta$ for $\widehat{d}=0.10 . D a=10^{-5}, \varepsilon=0.39$.

Figure 10. Critical solutal Rayleigh number versus the thermal Rayleigh number, for three values of the depth ratio $\widehat{d}$.

Figure 11. Wave number versus the thermal Rayleigh number, for three values of the depth ratio $\widehat{d}$.

Figure 12. Streamline patterns and vertical velocity profiles for $\widehat{d}=0.8$ : (a) $R a_{\mathrm{T}}=-20, R a_{\mathrm{S}}=-35.1$ and $\kappa_{c r}=10.2$; (b) $R a_{\mathrm{T}}=0, R a_{\mathrm{S}}=0$ and $\kappa_{c r}=6.0$; (c) $R a_{\mathrm{T}}=20, R a_{\mathrm{S}}=24.7$ and $\kappa_{c r}=3.5$; (d) $R a_{\mathrm{T}}=50, R a_{\mathrm{S}}=38$ and $\kappa_{c r}=4.0$. The thick horizontal line represents the fluid/porous interface.

Figure 13. Influence of the thermal diffusivity ratio $\varepsilon_{T}$ for $\widehat{d}=0.8$ and different thermal Rayleigh numbers.

\section{Figure Captions}

Table 1. Minimum values of the Rayleigh number for the "fluid mode". $D a=10^{-5}, \varepsilon=0.39$. 


\begin{tabular}{c|c|c|c|c|}
\cline { 2 - 5 } & \multicolumn{2}{c|}{$\widehat{d}=0.10$} & \multicolumn{2}{c|}{$\widehat{d}=0.14$} \\
\cline { 2 - 5 } & $-R a_{T c r}$ & $\kappa_{c r}$ & $-R a_{T c r}$ & $\kappa_{c r}$ \\
\hline \hline$\beta=0$ & 132.98 & 29.0 & 43.48 & 22.0 \\
$\beta=1$ & 121.77 & 28.0 & 40.48 & 22.0 \\
$\beta=1.5$ & 110.68 & 27.0 & 37.16 & 21.0 \\
$\beta=2$ & 88.20 & 24.0 & 29.18 & 19.0 \\
\hline
\end{tabular}

TABLE 1. Minimum values of the Rayleigh number for the "fluid mode". $D a=10^{-5}, \varepsilon=0.39$. 


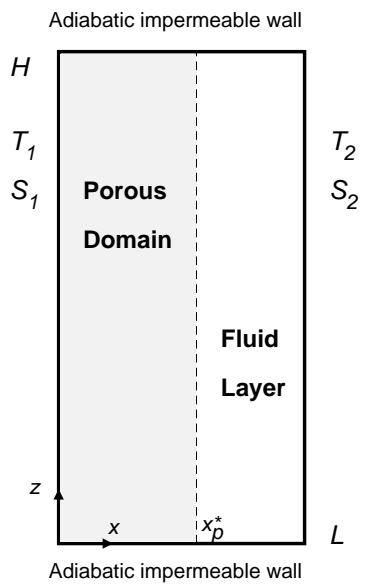

FIGURE 1. Schematic description of the problem. 


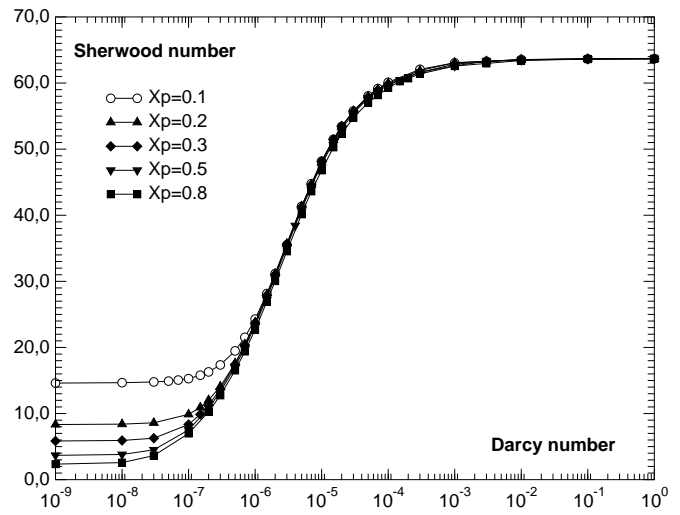

FIGURE 2. Mass transfer variation with permeability and porous layer thickness $\left(R a_{T}=10^{6}-N=10-\operatorname{Pr}=10-A=2\right)$ 


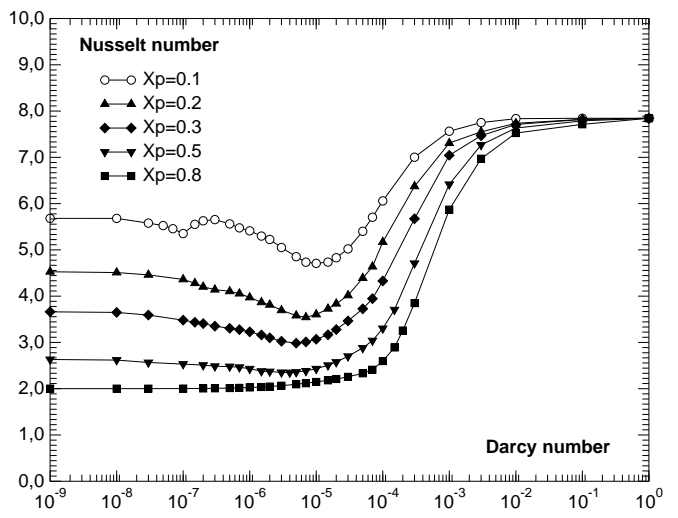

FIGURE 3. Heat transfer variation with permeability and porous layer thickness $\left(R a_{T}=10^{6}-N=10-\operatorname{Pr}=10-A=2-L e=100\right)$ 

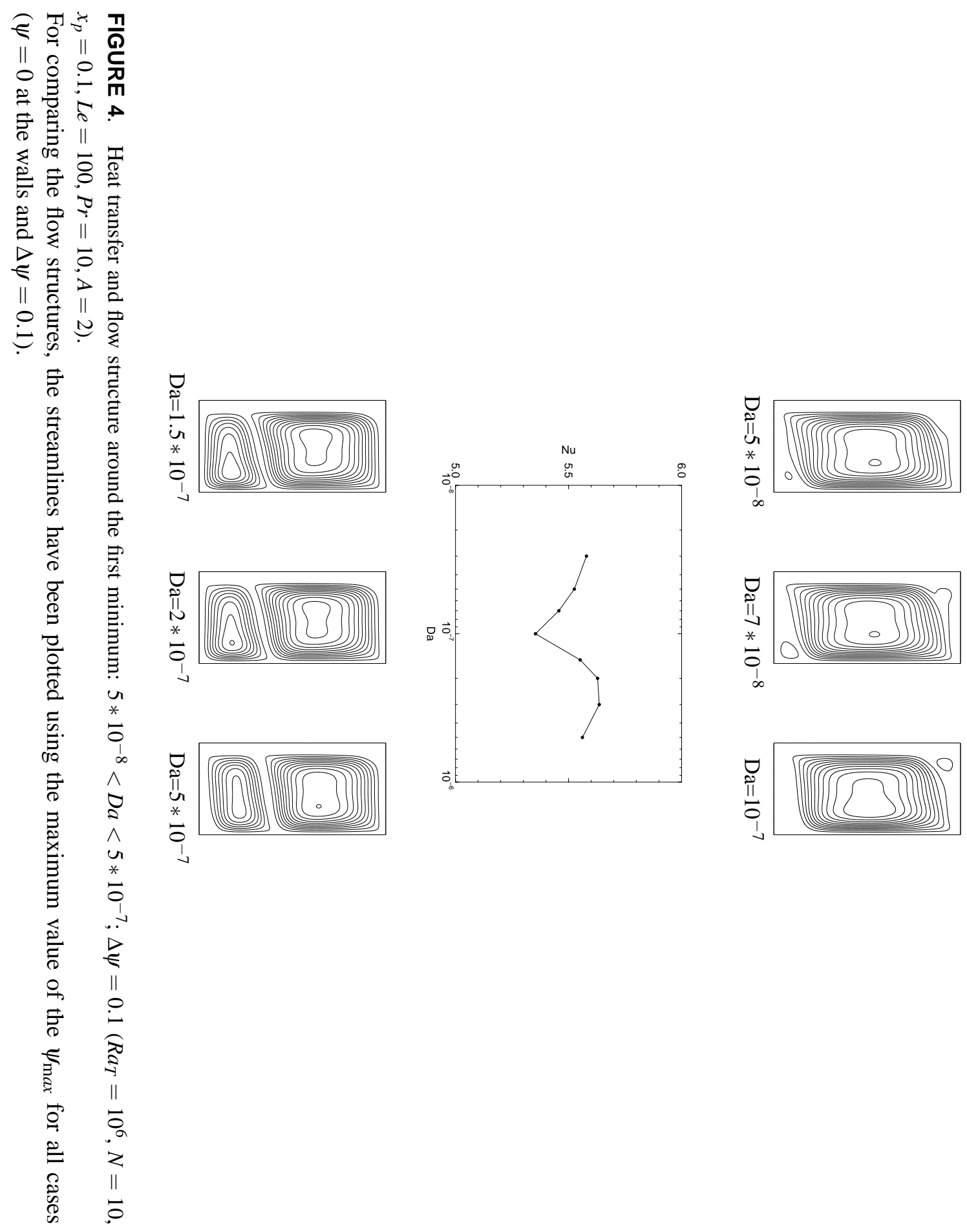


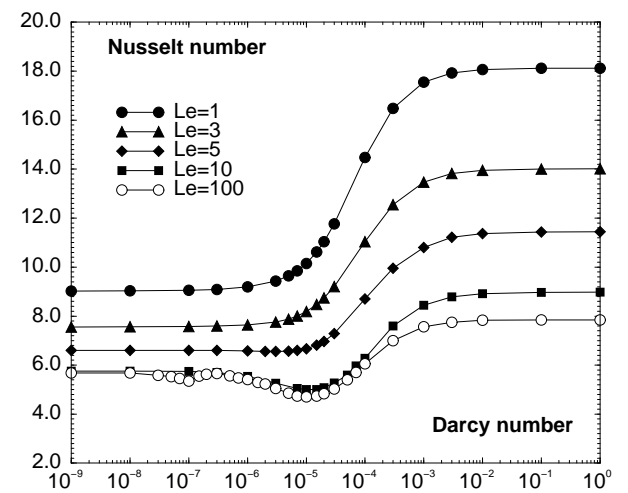

FIGURE 5. Heat transfer variation with permeability for different Lewis numbers $\left(R a_{T}=10^{6}-N=10-x_{\mathrm{P}}=0.1-\operatorname{Pr}=10-A=2\right)$ 


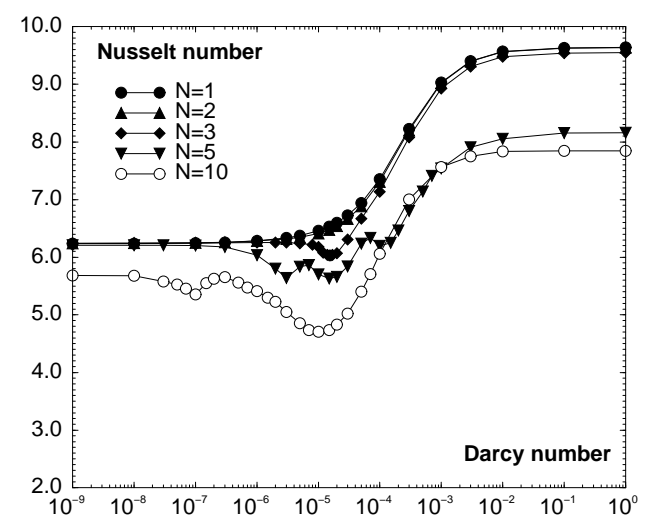

FIGURE 6. Heat transfer variation with permeability for different buoyancy ratios $\left(R a_{T}=10^{6}-x_{\mathrm{P}}=0.1-\operatorname{Pr}=10-L e=100-A=2\right)$ 


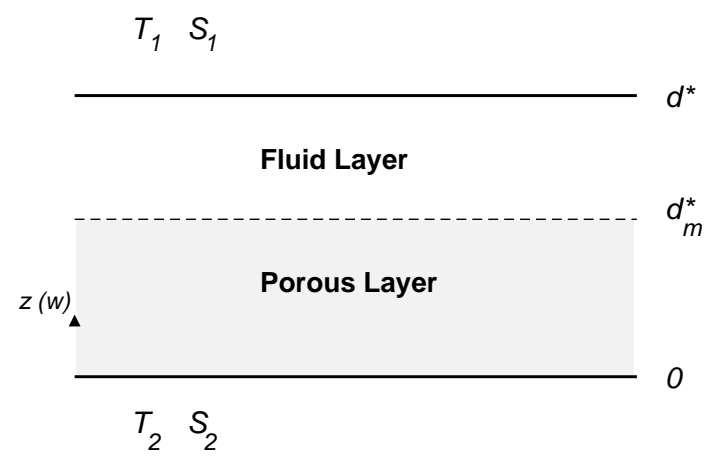

FIGURE 7. Geometric configuration of the problem. 


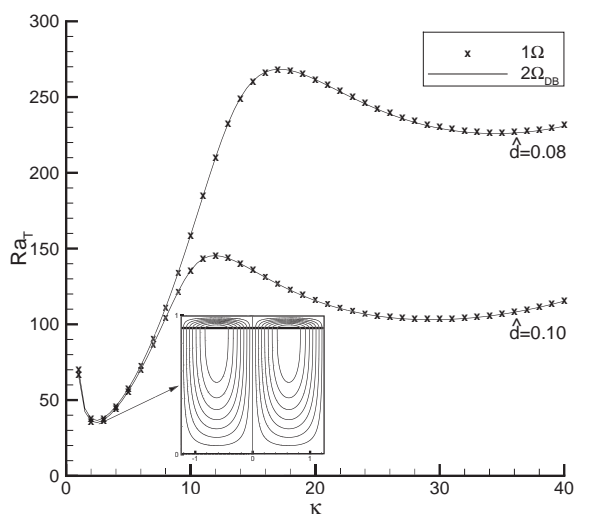

FIGURE 8. Marginal stability curves : comparison between the $1 \Omega$ and the $2 \Omega_{D B}$ approaches for $\widehat{d}=d_{f}^{*} / d_{m}^{*}=0.08$ and $\widehat{d}=0.10$ (Da $=7.4410^{-6}$. 


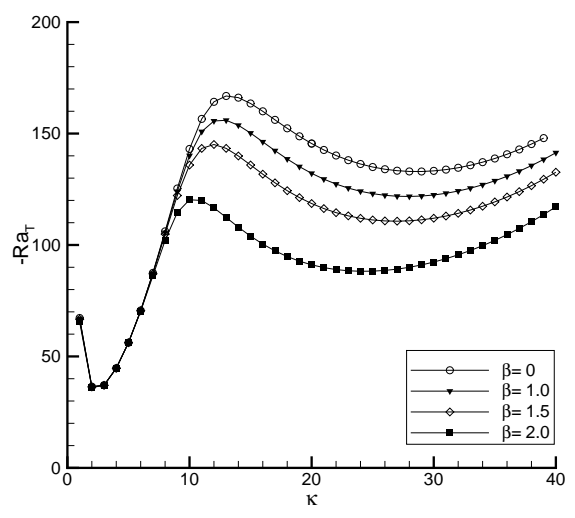

FIGURE 9. Influence of the stress jump coefficient $\beta$ for $\widehat{d}=0.10 . D a=10^{-5}, \varepsilon=0.39$. 


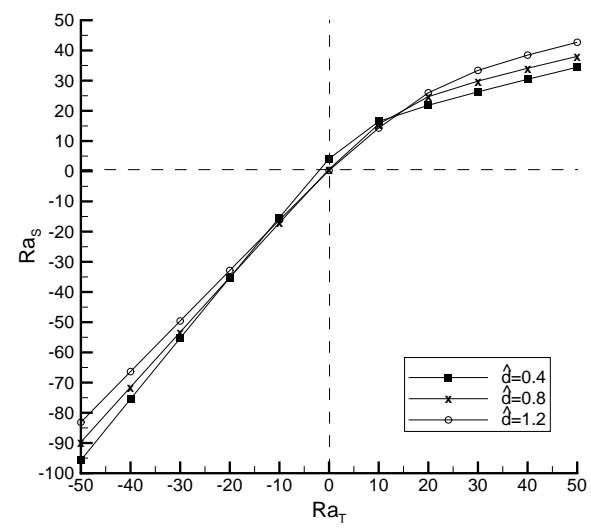

FIGURE 10. Critical solutal Rayleigh number versus the thermal Rayleigh number, for three values of the depth ratio $\widehat{d}$. 


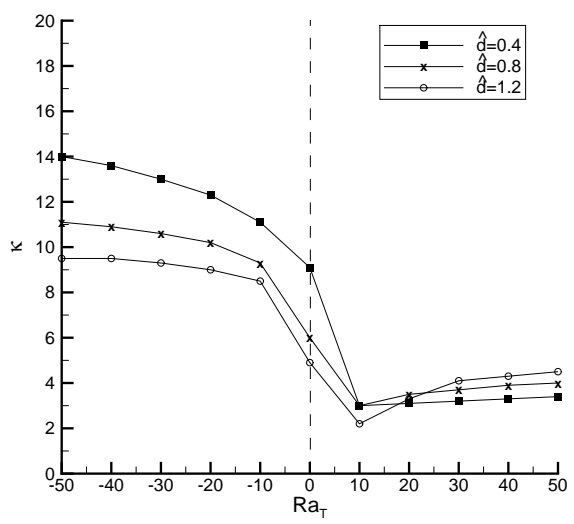

FIGURE 11. Wave number versus the thermal Rayleigh number, for three values of the depth ratio $\widehat{d}$. 


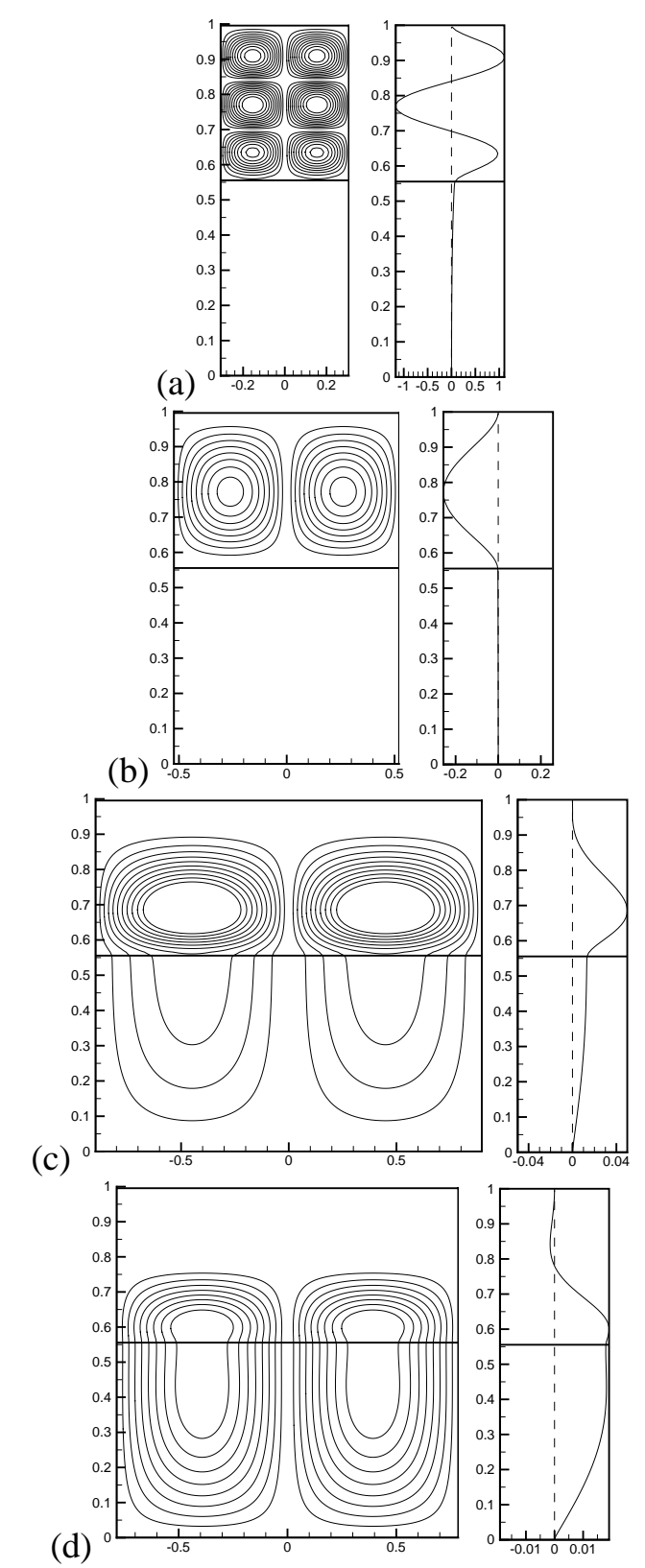

FIGURE 12. Streamline patterns and vertical velocity profiles for $\widehat{d}=0.8$ : (a) $R a_{\mathrm{T}}=-20, R a_{\mathrm{S}}=-35.1$ and $\kappa_{c r}=10.2$; (b) $R a_{\mathrm{T}}=0$, $R a_{\mathrm{S}}=0$ and $\kappa_{c r}=6.0$; (c) $R a_{\mathrm{T}}=20, R a_{\mathrm{S}}=24.7$ and $\kappa_{c r}=3.5$; (d) $R a_{\mathrm{T}}=50, R a_{\mathrm{S}}=38$ and $\kappa_{c r}=4.0$. The thick horizontal line represents the fluid/porous interface. 


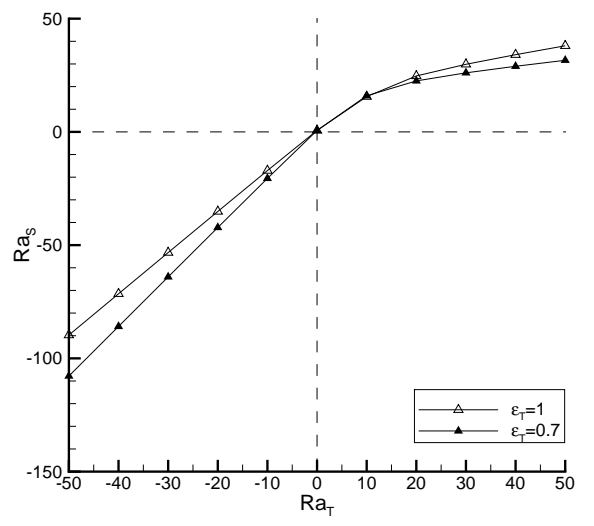

FIGURE 13. Influence of the thermal diffusivity ratio $\varepsilon_{T}$ for $\widehat{d}=0.8$ and different thermal Rayleigh numbers. 